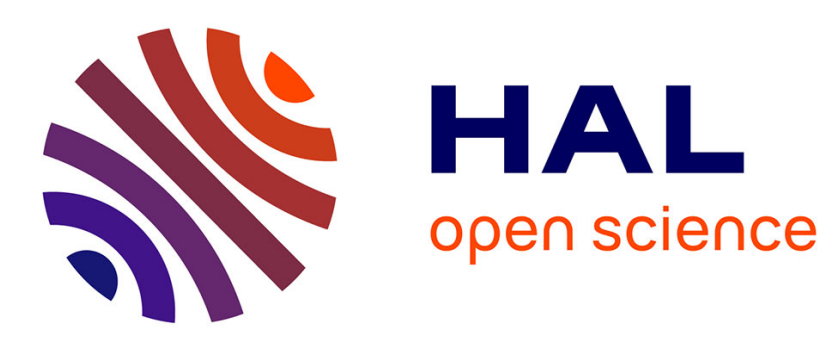

\title{
Application of cylindrical correlation functions to anisotropic scattering from amorphous films
}

\author{
M. Regan, A. Bienenstock
}

\section{To cite this version:}

M. Regan, A. Bienenstock. Application of cylindrical correlation functions to anisotropic scattering from amorphous films. Journal de Physique IV Proceedings, 1993, 03 (C8), pp.C8-459-C8-462. 10.1051/jp4:1993895 . jpa-00252326

\section{HAL Id: jpa-00252326 https://hal.science/jpa-00252326}

Submitted on 1 Jan 1993

HAL is a multi-disciplinary open access archive for the deposit and dissemination of scientific research documents, whether they are published or not. The documents may come from teaching and research institutions in France or abroad, or from public or private research centers.
L'archive ouverte pluridisciplinaire HAL, est destinée au dépôt et à la diffusion de documents scientifiques de niveau recherche, publiés ou non, émanant des établissements d'enseignement et de recherche français ou étrangers, des laboratoires publics ou privés. 


\title{
Application of cylindrical correlation functions to anisotropic scattering from amorphous films
}

\author{
M.J. REGAN and A. BIENENSTOCK
}

Department of Applied Physics, Stanford University, Stanford, CA 94305, U.S.A.

Stanford Synchrotron Radiation Laboratory, Bin 69, P.O. Box 4349, Stanford, CA 94309, U.S.A.

The cylindrical pair correlation function has been utilized as a structural tool in the study of anisotropic phase separation in magnetron sputtered amorphous $\mathrm{Fe}_{\mathrm{x}} \mathrm{Ge} 100-\mathrm{x}$ films. Anomalous small-angle $\mathrm{x}$-ray scattering (ASAXS) measurements have been performed with the scattering vector both in and oblique to the plane of the films, to search for anisotropy. The cylindrical correlation function has been calculated from these measurements and indicates that there exist well-defined regions of electron density that are elongated $(7 \AA$ by $12 \AA$ ) in the direction of film growth. In addition, for a composition of $x=6$, these regions are well-correlated on a length scale of $27 \AA$ for those correlation vectors oriented in the plane of the film surface, whereas the correlations are far weaker and longer in length for those vectors aligned in the direction of growth. These results are in agreement with a previous qualitative model of packed, prolate ellipsoids oriented in the direction of growth.

\section{Introduction}

A rather straightforward structural analysis of most amorphous alloys employs the Debye equation for the interpretation of $x$-ray scattering results, where correlations exist with equal probability for all orientations in space[1]. It is not obvious that vapor deposited amorphous alloys should exhibit this isotropy, however, since they are grown in one particular direction with their lateral dimensions fixed. We have shown that composition fluctuations that have been reported[2] in magnetron sputtered amorphous $\mathrm{Fe}_{\mathrm{x}} \mathrm{Ge}_{100-\mathrm{x}}$ films are quite anisotropic, depending significantly on the orientation of the scattering vector with respect to the sample normal[3]. The previous work utilized anomalous scattering to show that phase separation occurs into regions of a-Ge and an amorphous $\mathrm{Fe}-\mathrm{Ge}$ intermetallic, likely a-FeGe 2 . Since real-space information is contained in the fourier transform of the scattering projected along the different directions of interest in real space, it is difficult to interpret the anisotropy from the scattering patterns alone. Any robust understanding, especially of an anisotropic noncrystalline system, thus relies on determining the electron density pair correlation function. From the vapor deposition process itself, the amorphous alloy is likely to exhibit an "in-plane" isotropy with an axis of cylindrical symmetry in the direction of growth. The appropriate function in this case is the cylindrically symmetric pair correlation function[4]. Such functions have been used to study oriented noncrystalline polymers[5], but to our knowledge have not been applied to vapor-deposited amorphous semiconductors or metals.

\section{Theory}

The electron density pair correlation function, $\gamma(\bar{r})$, is the average over all two-point correlations of the deviation in electron density from the average[6]. It is the Fourier transform of the observed $x$-ray scattering function, the structure factor $S(\vec{k})$. For a cylindrical correlation function, Norman[4] has shown it possible to expand the $\gamma(\vec{r})$ and $S(\tilde{k})$ in terms of Legendre polynomials,

$$
\gamma\left(r, \theta_{r}\right)=\sum_{n=0,2,4 . .}^{\infty} P_{n}\left(\cos \theta_{r}\right) \gamma_{n}(r) \text { and } S\left(k, \theta_{k}\right)=\sum_{n=0,2,4 .}^{\infty} P_{n}\left(\cos \theta_{k}\right) S_{n}(k),
$$

where the coefficients $\gamma_{n}(r)$ and $S_{n}(k)$ satisfy the spherical Bessel transform

$$
\gamma_{n}(r)=\frac{i^{n}}{2 \pi^{2}} \int_{0}^{\infty} k^{2} j_{n}(k r) S_{n}(k) d k
$$


and $\left(r, \theta_{r}, \phi_{r}\right)$ and $\left(k, \theta_{k} \phi_{k}\right)$ are spherical polar coordinates in real and reciprocal space that describe $\gamma(\vec{r})$ (figure 1a). Due to the cylindrical symmetry, $\phi_{r}$ and $\phi_{k}$ do not enter into the final relations and are dropped from discussion. The $S_{n}(k)$ are readily obtained by the orthogonality relations, i.e.

$$
S_{n}(k)=(2 n+1) \int_{0}^{\pi / 2} S\left(k, \theta_{k}\right) P_{n}\left(\cos \theta_{k}\right) \sin \theta_{k} d \theta_{k}
$$

Only the even terms in $n$ are of interest, since we simplify the problem and assume that there exists an inversion symmetry with respect to the $x-y$ or $k_{x}-k_{y}$ plane. This need not be the case, however, as one can imagine a situation where correlations in the direction of film growth $+\hat{z}$ differ from those in the $-\hat{z}$ direction[7].

Legendre polynomials form a complete set, but it is not possible to uniquely determine all $S_{n}(k)$ from the finite data collected. For example, if there exist nine different radial scans in $k$ that span $\theta_{k}=0^{\circ}$ to $90^{\circ}$, the largest $n$ that can be determined is eight (the highest order polynomial one expects to fit nine data points is of order eight). Hence, large regions of reciprocal space must be sampled in order to approximate $S\left(k, \theta_{k}\right)$ as a Legendre series. This is not trivial. Only those samples that lack long-range order and exhibit slight anisotropy are amenable to this method.

(a)

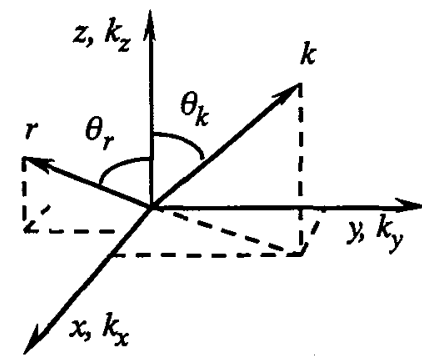

(b)

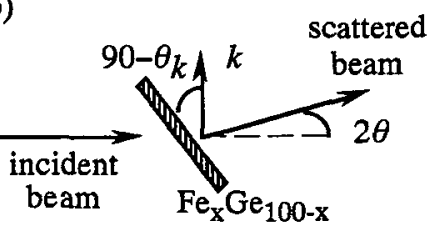

Figure 1. (a) Polar coordinate system for cylindrical correlation function. (b) Scattering geometry for oblique transmission experiment.

\section{Experiment}

Amorphous $\mathrm{Fe}_{\mathrm{x}} \mathrm{Ge}_{100-\mathrm{x}}$ films were prepared by magnetron co-sputtering of elemental targets onto $\mathrm{Si}$ wafers attached to a rapidly rotating substrate table. The Si substrates were then removed and the films ( 6-9 microns thick) rendered free-standing after immersion in a warm KOH bath[7]. Initial characterization of the samples included a check for crystallization with a conventional Picker diffractometer, determination of the composition with an electron microprobe and a thickness measurement with an alpha-step profilometer.

The SAXS experiment was performed on the eight-pole focused wiggler end-station 4-2 at the Stanford Synchrotron Radiation Laboratory. The basic line consists of a bent cylindrical mirror and a double-crystal monochromator. In order to test for anisotropy, oblique transmission measurements[8] which allow for various orientations of the scattering vector were performed. By tilting the sample to angle $\theta_{k}=90,75,60,45,30$, and $15^{\circ}$, the scattering vector rotates out of the surface plane, and correlations in electron density are projected onto the plane defined at angle $90-\theta_{k}$ with respect to the sample surface plane $\left(\theta_{k}=90^{\circ}\right)$--see figure $1 \mathrm{~b}$. The data were background-subtracted, scaled per irradiated volume and absorption, and placed on an absolute scale. We report results in terms of the structure factor, $S(\bar{k})$, equal to the differential cross-section per unit volume scaled by the classical radius of the electron $\left(r_{e}\right)$ squared, with $k=4 \pi \sin \theta / \lambda$. The details of the data collection, normalization, and manipulation procedures will be summarized more completely by Regan[3].

In contrast to atomic pair correlation functions used in studies of short-range order, we have not attempted to remove the "independent scattering," since it does not appear obvious how to remove the self-scattering contribution from SAXS data. Indeed, for the case of large interparticle interference, it is still not possible to remove the contribution from the particles' self-correlations since there exists a 
distribution in particle size as well as particle interpenetration. We thus present the cylindrical pair correlation function which includes the self-scattering contribution. The number of vectors that give an identical correlation between $r$ and $r+d r$ and $\theta_{r}$ and $\theta_{r}+d \theta_{r}$ is thus $4 \pi r^{2} \sin \theta_{r} r\left(r, \theta_{r}\right) d \theta_{r} d r$, with a factor of 2 included for inversion symmetry.

\section{Analysis and Results}

Figure $2 \mathrm{a}$ illustrates the structure factor $S\left(k, \theta_{k}\right)$ determined from the oblique transmission experiment for $\mathrm{a}-\mathrm{Fe}_{6} \mathrm{Ge}_{94}$ at $6912 \mathrm{eV}$. A strong anisotropy appears, observable from the change in the scattering peak with sample orientation. As the sample is tilted, the peak shifts inward to smaller $k$, the intensity monotonically decreasing as the scattering vector becomes more aligned with $k_{z}$. These data indicate that there exist fluctuations in electron density which become gradually weaker and of increasingly greater distance as the scattering vector shifts in orientation from the sample surface to sample normal. The other samples in this composition range $(x<33)$ illustrate the same behavior. That is, there is a monotonic decrease in scattering intensity and shift inward in peak position with changes in $\theta_{k}$. Hence, we choose the $x=6$ sample as representative of the $x<33$ alloy and examine in close detail its cylindrical correlation function.

(a)

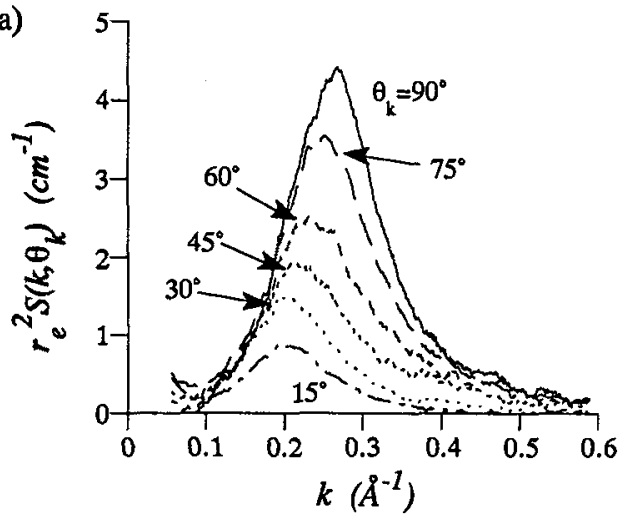

(b)

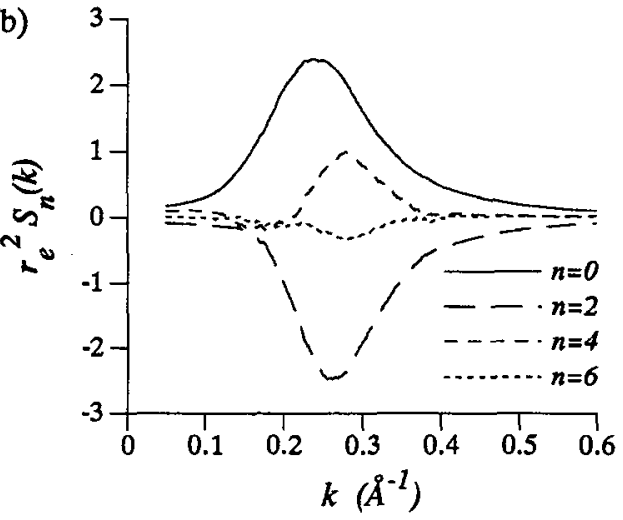

Figure 2. (a) Oblique transmission SAXS experiment for $\mathrm{x}=6$ sample. (b) Legendre coefficients $S_{n}(k)$.

The anisotropic scattering data have been used to determine the first four $S_{n}(k)$ from Eq. 3 by employing a 7-point Simpson's rule. For each $k$, there are six measured points $\theta_{k}=90,75,60,45,30$, and $15^{\circ}$. The seventh is not measurable but not necessary to compute $S_{n}(k)$, since the factor of $\sin \theta_{k}$ in the integrand forces the $\theta_{k}=0^{\circ}$ term of the total integrand to 0 . Figure $2 \mathrm{~b}$ shows the computed $S_{n}(k)$. The $n=2$ and $n=4$ terms are far from negligible and illustrate the importance of including the anisotropy. The $n=6$ term, however, is much weaker, contributing far less to the series. The $S\left(k, \theta_{k}\right)$ computed from the $S_{n}(k)$ provides an approximation of better than $2 \%$ to the raw data, except for the $\theta_{k}=90^{\circ}$ scan near the SAXS maximum, where the greatest anisotropy is observed and the fit is no worse than $10 \%$, a result of not including higher order $S_{n}(k)$ or, consequently, an inadequate sampling of reciprocal space.

The spherical Bessel transforms of the $S_{n}(k)$ have been computed and the corresponding Legendre series summed to obtain the cylindrical correlation function (figure 3 ). The large maximum near $r=0$ is from regions of electron density correlated with themselves. That is, regions that are large enough to define a local electron density but small enough so that variations in density within are not observed. Correlations for $r<10 \AA$ also exhibit a large, positive $\gamma\left(r, \theta_{r}\right)$, due to the similarity of electron density of neighboring regions and are often considered the result of a "particle" of certain dimensions of a particular electron density. In this case, the dimensions of the particle appear elongated in the $z$ direction by more than a factor of 3 to 2 (table 1 ).

Beyond the contribution from self-correlation $(r>10 \AA)$, oscillations in $\gamma\left(r, \theta_{r}\right)$ about 0 are present. Table 1 shows the secondary maxima for a few $\theta_{r}$, which illustrate the strong oscillations in amplitude present for correlations in the plane of the sample. For those along $\theta_{r}=45^{\circ}$, the oscillations begin to wane in magnitude and frequency. Finally, for a correlation vector in the direction of film growth, oscillations are not obvious but exist at much greater distance than in the previous cases and at much 
smaller magnitude. These results are consistent with a previous model of close-packed homogeneous ellipsoids with a Born-Green interparticle interference function. For the $x=6$ composition, the model consists of a-FeGe 2 ellipsoids in a background of a-Ge. The ellipsoids are of approximate diameter $\{7$, $7,13\} \AA$ packed with a hard-core interparticle potential such that the center of one ellipsoid can approach no closer than $\{21,21,30\} \AA$ of the center of an another. By applying a cylindrical correlation function analysis, we have a more direct and satisfying approach to the anisotropy, especially since the model assumes particle homogeneity, monodispersity, and an equilibrium liquid structure factor to explain interparticle interference in the vapor-deposited state.

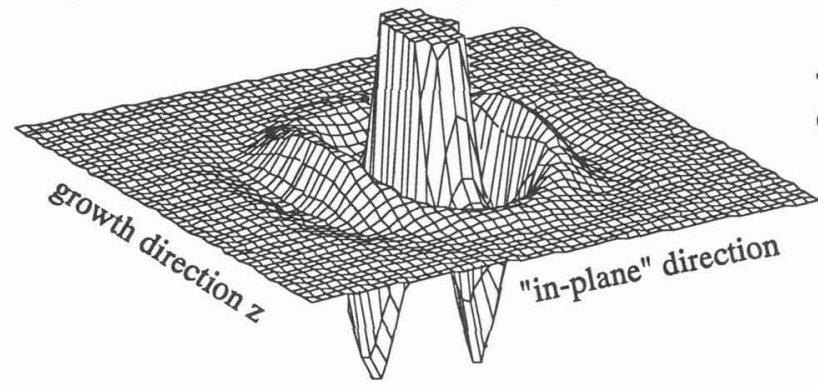

Table 1. Correlation parameters for different film directions.

\begin{tabular}{c|c|c}
$\theta_{r}\left(^{\circ}\right)$ & $\begin{array}{c}\text { FWHM } \\
r=0(\AA)\end{array}$ & $\begin{array}{c}\text { secondary } \\
\text { maxima }(\AA)\end{array}$ \\
\hline $90(\mathrm{x}-\mathrm{y})$ & 7.3 & $26.6,50.0,76.3$ \\
45 & 9.0 & $32.4,64.8$ \\
$0(\mathrm{z})$ & 11.7 & 41
\end{tabular}

Figure 3. Three-dimensional mesh plot of $\gamma(\vec{r})$, where $\gamma(\vec{r})$ for small $r$ has been truncated in order to better observe the oscillations. Mesh lines are spaced $3 \AA$ by $3 \AA$.

\section{Conclusions}

Vapor-deposited a- $\mathrm{Fe}_{\mathrm{x}} \mathrm{Ge}_{100-\mathrm{x}}(\mathrm{x}<33)$ films exhibit a cylindrical symmetry, with the axis of symmetry in the direction of film growth. Although it is not obvious at this point as to the origin of the anisotropic structure, the cylindrical correlation function analysis provides a powerful tool to view the anisotropic structure in real space. With ample scans in $k$-space to adequately sample the anisotropy, the cylindrical correlation function can be obtained at little computational cost. Results are consistent with a previous model of phase separation into a collection of oriented prolate ellipsoids of a-FeGe 2 in a background of a-Ge.

\section{Acknowledgements}

This research was performed at and supported by the Stanford Synchrotron Radiation Laboratory, which is funded by the Department of Energy through the Office of Basic Energy Science and the NIH, Biotechnology Resource Program, Division of Research Resources. The authors would like to thank P. Lecante for assistance with data collection and Th. Gerber and B. Himmel for suggestions concerning isotropic pair correlation functions.

\section{References}

[1] See, for example, B.E. Warren, X-Ray Diffraction, (Addison-Wesley Publishing, Reading, 1969).

2] M. Rice, S. Wakatsuki, and A. Bienenstock, J. Appl. Cryst. 24, 598 (1991).

[3] M.J. Regan and A. Bienenstock, submitted to Mater. Res. Soc. M.J. Regan, PhD thesis, in preparation, Stanford University.

[4] N. Norman, PhD thesis, University of Oslo, 1954.

[5] M.E. Milberg, J. Appl. Phys. 34, 722 (1962).

6] O. Glatter and O. Kratky, Small-Angle X-ray Scattering, (Academic Press, London, 1982).

77 L.C. Wilson, PhD thesis, Stanford University, 1990.

[8] G.S. Cargill, Phys. Rev. Lett. 28, 1372 (1972). 